Portland State University

PDXScholar

\title{
Anomalous low-temperature and high-field magnetoresistance in the organic superconductor $\boldsymbol{\beta}$ "-(BEDT-TTF)2SF5CH2CF2SO3
}

F. Zuo

X. Su

P. Zhang

J. S. Brooks

J. Wosnitza

See next page for additional authors

Follow this and additional works at: https://pdxscholar.library.pdx.edu/chem_fac

Part of the Physics Commons

Let us know how access to this document benefits you.

\section{Citation Details}

Zuo, F., Su, X., Zhang, P., Brooks, J. S., Wosnitza, J., Schlueter, J. A., Williams, Jack M., Nixon, P. G., Winter, R. W., Gard, G. L. (1999). Anomalous low-temperature and high-field magnetoresistance in the organic superconductor $\beta "$ "-(BEDT-TTF) ${ }_{2} \mathrm{SF}_{5} \mathrm{CH}_{2} \mathrm{CF}_{2} \mathrm{SO}_{3}$. Physical Review B., 606296.

This Article is brought to you for free and open access. It has been accepted for inclusion in Chemistry Faculty Publications and Presentations by an authorized administrator of PDXScholar. Please contact us if we can make this document more accessible: pdxscholar@pdx.edu. 


\section{Authors}

F. Zuo, X. Su, P. Zhang, J. S. Brooks, J. Wosnitza, J. A. Schlueter, Jack M. Williams, P. G. Nixon, Rolf Walter Winter, and Gary L. Gard 


\title{
Anomalous low-temperature and high-field magnetoresistance in the organic superconductor $\beta^{\prime \prime}$-(BEDT-TTF) ${ }_{2} \mathrm{SF}_{5} \mathrm{CH}_{2} \mathrm{CF}_{2} \mathrm{SO}_{3}$
}

\author{
F. Zuo, X. Su, and P. Zhang \\ Department of Physics, University of Miami, Coral Gables, Florida 33124 \\ J. S. Brooks \\ National High Magnetic Field Laboratory, Tallahassee, Florida 32306 \\ J. Wosnitza \\ Physikalisches Institut, Universität Karlsruhe, D-76128 Karlsruhe, Germany \\ J. A. Schlueter and Jack M. Williams \\ Chemistry and Materials Science Divisions, Argonne National Laboratory, Argonne, Illinois 60439 \\ P. G. Nixon, R. W. Winter, and G. L. Gard \\ Department of Chemistry, Portland State University, Portland, Oregon 97207 \\ (Received 24 November 1998; revised manuscript received 1 March 1999)
}

\begin{abstract}
We report direct observations of anomalous magnetic-field and temperature dependences of the Shubnikov-de Haas oscillations in the organic superconductor $\beta^{\prime \prime}$-(BEDT-TTF) ${ }_{2} \mathrm{SF}_{5} \mathrm{CH}_{2} \mathrm{CF}_{2} \mathrm{SO}_{3}$. Unlike other BEDT-TTF based organic superconductors, a nonmetallic temperature dependence of the background magnetoresistance is clearly observed. It is speculated that the nonmetallic behavior may arise from a partial nesting of the open orbits, similar to the field-induced density wave in the quasi-one-dimensional systems or a charge localization. The analysis of the magnetoresistance oscillations are found to deviate from the conventional Lifshitz-Kosevich description at high field and low temperatures. [S0163-1829(99)05433-8]
\end{abstract}

BEDT-TTF [bis(ethylenedithio)tetrathiafulvalene] based organic superconductors have been of great interest since their discovery in early 1980's. The charge transfer salts (BEDT-TTF) ${ }_{2} X$ consist of alternating layers of BEDT-TTF molecules (electron donors) and $X$ molecules (electron acceptors). The layered structure plus a typically simple Fermi surface make them ideal candidates to study the lowtemperature electronic properties via the de Haas-van Alphen (dHvA) and the Shubnikov-de Haas effect (SdH). Much experimental effort has been devoted to such studies in the organic superconductors to correlate with band structure calculations. ${ }^{1-9}$

In this paper, we report direct experimental evidence of a possible field induced metal-insulator transition at low temperatures in the recently discovered all organic superconductor $\beta^{\prime \prime}$-(BEDT-TTF) ${ }_{2} \mathrm{SF}_{5} \mathrm{CH}_{2} \mathrm{CF}_{2} \mathrm{SO}_{3}$. Resistance measurements at low temperatures show a nonmetallic temperature dependence of the background magnetoresistance. This is in sharp contrast with other known organic metals where the background resistance in the $\mathrm{SdH}$ oscillation decreases or saturates with decreasing temperature. It is speculated that this might be due to a formation of a fieldinduced density wave of the open bands at low temperatures and high fields or a possible field induced localization of charges carriers. Analysis of the $\mathrm{SdH}$ oscillation shows clear deviation from the three dimensional (3D) Lifshitz-Kosevich theory at low temperatures and high fields.

Single crystals of $\beta^{\prime \prime}$-(BEDT-TTF) ${ }_{2} \mathrm{SF}_{5} \mathrm{CH}_{2} \mathrm{CF}_{2} \mathrm{SO}_{3}$ were synthesized by a electrocrystallization technique described elsewhere. ${ }^{10}$ Several crystals were used in these measurements with average dimensions of $1 \times 0.7 \times 0.3 \mathrm{~mm}$, with superconducting transition temperatures $T_{c} \sim 5 \mathrm{~K}$. The
$T_{c}$ is defined as the midpoint in the resistive transition. The room temperature interlayer resistivity is about $700 \Omega \mathrm{cm}$ and the in-plane resistivity about $0.2 \Omega \mathrm{cm}$. The resistivity ratio between room temperature and just above superconducting transition temperature is $\rho_{\perp}(300 \mathrm{~K}) / \rho_{\perp}(6 \mathrm{~K})$ $\sim 230$. The resistance was measured with use of the four probe technique for both interlayer and in-plane resistivity. Contact of the gold wires to the sample was made with a Dupont conducting paste or graphite paste. Typical contact resistances between the gold wire and the sample were about $10 \Omega$. A current of $1 \mu \mathrm{A}$ was used to ensure linear $I-V$ characteristics. The voltage was detected with a lock-in amplifier at low frequencies of about $312 \mathrm{~Hz}$. To avoid pressure effects due to solidification of grease, the sample was mechanically held by thin gold wires. The data presented in this work are taken in a ${ }^{3} \mathrm{He}-{ }^{4} \mathrm{He}$ dilution refrigerator with field up to $17.8 \mathrm{~T}$. Magnetic field is applied perpendicular to the conducting planes.

Shown in Fig. 1 is an overlay of the interlayer magnetoresistance as a function of field at different temperatures $\mathrm{Ta}$ $=0.660 \mathrm{~K}, 0.565 \mathrm{~K}, 0.455 \mathrm{~K}, 0.218 \mathrm{~K}, 80 \mathrm{mK}$, and $47 \mathrm{mK}$. Toward the high field the curves shifts upward with decreasing temperature. At high temperatures, the interlayer magnetoresistance displays a peak as a function of field for $H$ $<3 T$. The peak disappears for $T \leqq 0.455 \mathrm{~K}$. Commencing at about $H=4 \mathrm{~T}$, SdH oscillations can be clearly observed with the oscillation frequency at $200 \mathrm{~T}$ or $\Delta(1 / H)$ $=0.05 \mathrm{~T}^{-1}$. The inset shows the extrapolation of the background magnetoresistance at high field. For each temperature, the peak (valley) field and resistance are picked from the 


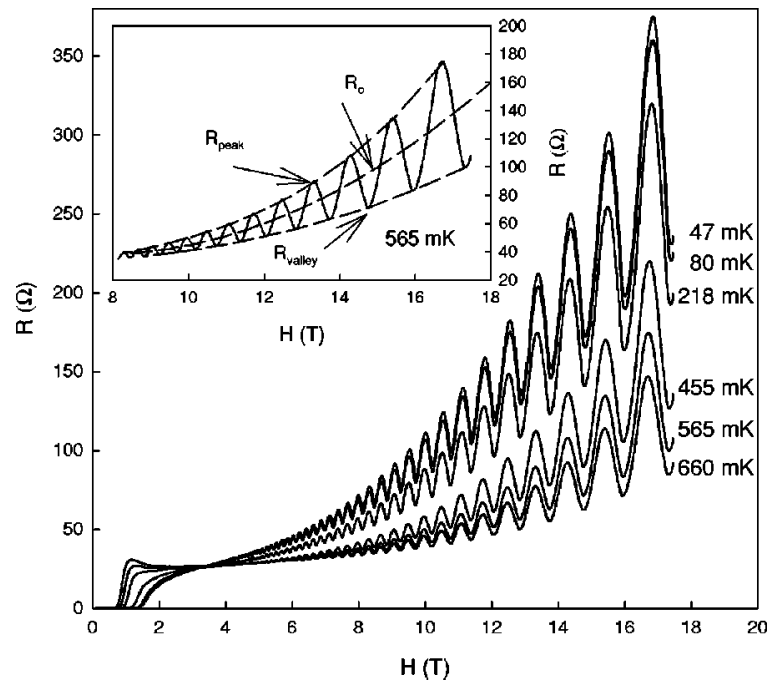

FIG. 1. Field dependence of the interlayer resistance at different temperatures. The inset shows the extrapolation of the background resistance.

experimental data and fitted to a simple quadratic expression $R_{\text {peak,valley }}(H)=a+b H+c H^{2}$, as shown by the envelopes. The background resistance $R_{0}$ is taken as the average of the two functions and the oscillation amplitude as the difference. Alternatively, the background magnetoresistance can also be obtained by a least square fit of the raw data over a limited field range. For a given field range, the two approaches give similar results. It should be mentioned that similar field and temperature dependences of magnetoresistance oscillations have been observed for the in-plane resistance, except that there is no magnetoresistance peak in the low-field region.

Shown in Fig. 2 is a plot of the background magnetoresistance as a function of temperature at various fields from 9 to $17 \mathrm{~T}$ with an increment of $1 \mathrm{~T}$. The curves shift upwards

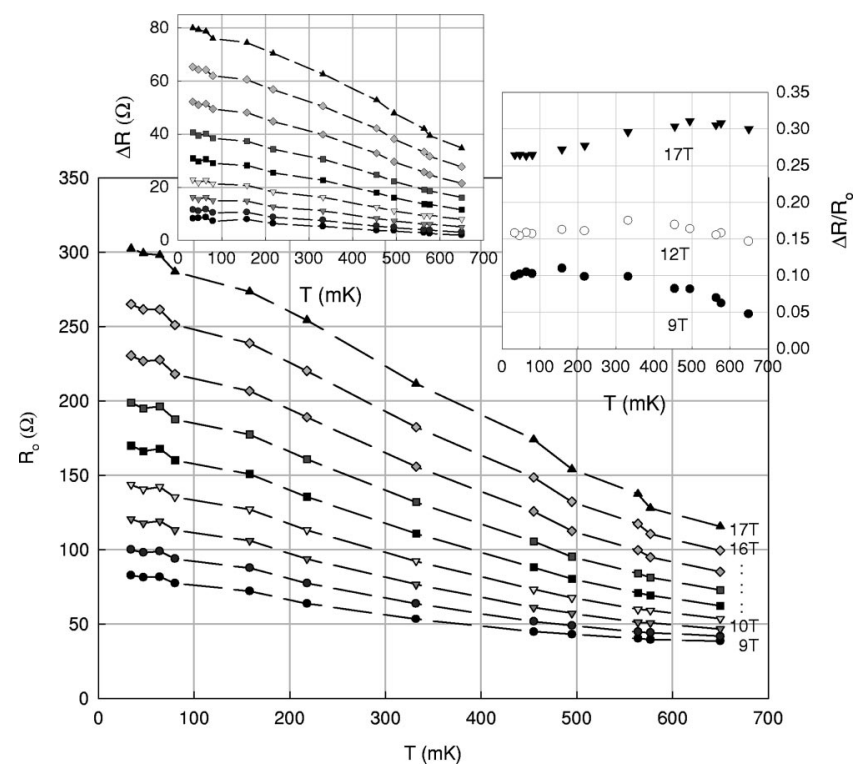

FIG. 2. Temperature dependence of the background magnetoresistance at different fields. The upper left inset shows the extrapolated peak amplitude as a function of $T$ and $H$. The upper right inset shows the relative change of resistance as a function of $T$ at $H=9$, 12 , and $17 \mathrm{~T}$. with increasing field. At a fixed field, the resistance increases with decreasing temperature and tends to saturate at very low temperatures. Shown in the upper right inset is the change of magnetoresistance $\Delta R=\left(R_{\text {peak }}-R_{\text {valley }}\right) / 2$ as a function of temeprature for the same fields as in Fig. 2. With decreasing temperature, both the oscillation amplitude $\Delta R$ and the background resistance increase. The upper right inset shows the relative change of the $\Delta R / R_{0}$ at three fields $H=9,12$, and $17 \mathrm{~T}$. Notice these fields are used for simplicity of analysis, they do not correspond to the experimental peak fields. With increasing field, $\Delta R / R_{0}$ shows increasingly stronger decrease at low temperatures.

The $\beta^{\prime \prime}$-(BEDT-TTF $)_{2} \mathrm{SF}_{5} \mathrm{CH}_{2} \mathrm{CF}_{2} \mathrm{SO}_{3}$ is to our knowledge the first BEDT-TTF based organic superconductors displaying an increasing background magnetoresistance with decreasing temperature. In the well studied $\kappa$-(BEDT-TTF $)_{2} \mathrm{Cu}(\mathrm{NCS})_{2}$ salt, ${ }^{1}$ the background magnetoresistance is relatively constant for temperature below $4 \mathrm{~K}$ and shows a tendency of saturation with field below $30 \mathrm{~T}$. Recent studies in the $\kappa$-(BEDT-TTF $)_{2} \mathrm{Cu}\left[\mathrm{N}(\mathrm{CN})_{2} \mathrm{Br}\right]$ salt shows a monotonic decrease of background magnetoresistance with decreasing temperature. ${ }^{7,8}$ Similar metallic temperature dependence of the background resistance has been found for $\kappa$-(BEDT-TTF $)_{2} \mathrm{I}_{3}{ }^{2}$ Although a somewhat similar semiconducting background magnetoresistance has been reported in the isostructural (BEDO-TTF) ${ }_{2} \mathrm{ReO}_{4} \cdot \mathrm{H}_{2} \mathrm{O}$ [bis(ethylenedioxy)tetrathiafulvalene], ${ }^{11}$ the latter compound undergoes a metal-insulator transition at around $35 \mathrm{~K}$ at ambient pressure. No transport anomaly has been observed from room temperature down to the superconducting transition in the present system. It should be noted that the nonmetallic temperature dependence of the background in the present compound is only observed at low temperatures and high fields. Resistance measurements at temperatures above $2 \mathrm{~K}$ show a metallic temperature dependence for fields up to 8 $\mathrm{T}^{12}$

Unlike the $\kappa$-(BEDT-TTF $)_{2} \mathrm{Cu}(\mathrm{NCS})_{2}$ salt, the $\mathrm{SdH}$ oscillation displays a clear deviation from the conventional Lifshitz-Kosevich (LK) description in its field and temperature dependence. ${ }^{13}$ Shown in Figs. 3(a) and 3(b) are overlays of the relative changes of the conductance $\Delta \sigma / \sigma$ as a function of field at different temperatures $T=0.65,0.45,0.22$, and $0.047 \mathrm{~K}$, here $\Delta \sigma / \sigma=\left[1 / R(H)-1 / R_{0}(H)\right] /\left[1 / R_{0}(H)\right]$ $=R_{0}(H) / R(H)-1 . R_{0}(H)$ is the background resistance extrapolated from a least square fit for the specified field range. At low field $(6 \mathrm{~T}<H<8 \mathrm{~T})$ as shown in Fig. 3(a), $\Delta \sigma / \sigma$ increases with decreasing temperature, in accord with the LK theory for a three-dimensional (3D) metals. By treating the relative change in conductivity as having the same temperature and field dependence as the magnetization, one can write

$$
\frac{\Delta \sigma}{\sigma} \propto \frac{T \exp \left(-\alpha \mu_{c} T_{D} / H\right)}{\sqrt{H} \sinh \left(\alpha \mu_{c} T / H\right)} \sin (2 \pi F / H+\gamma) .
$$

Here $\alpha=2 \pi^{2} k_{B} m_{e} / e \hbar=14.69 \mathrm{~T} / \mathrm{K}, \quad \mu_{c}=m_{c} / m_{e}$, the relative cyclotron mass. The oscillation frequency is given by $F=(\hbar / 2 \pi e) S$ with $S$ being the extremal cross section of the Fermi surface. $T_{D}$ is the Dingle temperature defined as $\hbar / 2 \pi k_{B} \tau, \tau$ is the electron scattering time. For constant $H$, the $\Delta \sigma / \sigma$ increases with decreasing $T$ and saturates in the $T \rightarrow 0$ limit. The expression has been used extensively in mapping out the Fermi surface in the organic metals and an 

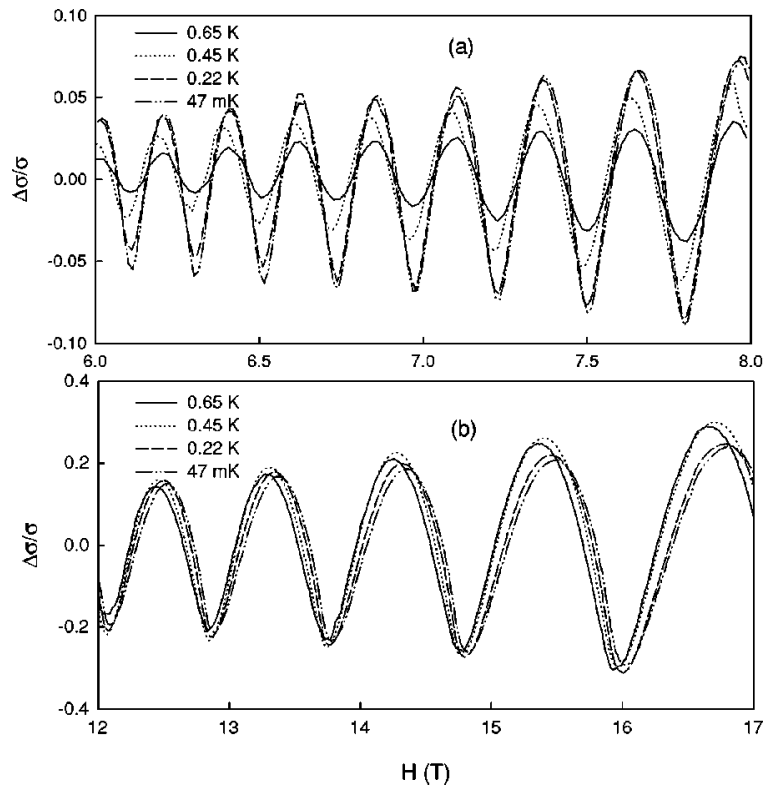

FIG. 3. (a) Field dependence (6-8 T) of the relative conductance at different temperatures. (b) Field dependence (12-17 T) of the relative conductance at different temperatures.

overall excellent agreement with direct magnetic dHvA measurements has been found. ${ }^{9}$

Figure 3(b) shows the high field $(12 \mathrm{~T}<\mathrm{H}<17 \mathrm{~T})$ dependence of $\Delta \sigma / \sigma$ for the same temperatures as in Fig. 3(a). However, unlike in the previous case, $\Delta \sigma / \sigma$ decreases with decreasing temperatures, especially in the high field end. For example, the peak amplitude is over $20 \%$ smaller at $47 \mathrm{mK}$ than at $0.45 \mathrm{~K}$ for $H$ between 16 and $17 \mathrm{~T}$. This is in sharp contrast with the LK theory.

Shown in Fig. 4 is a plot of the FFT amplitude of $\Delta \sigma / \sigma$ as a function of temperature at different fields $H=6,9$, and $12 \mathrm{~T}$. The solid lines are fits to Eq. (1). Clearly, a good fit is obtained for $H=6 \mathrm{~T}$. The fit gives an effective mass of $2.0 \pm 0.1 m_{e}$. At higher fields $H=9$ and $12 \mathrm{~T}$, only points at high temperatures can be fit. The fits give the same $\mu_{c}$ $=2.0 \pm 0.1$ with the curves deviating from the data at corre-

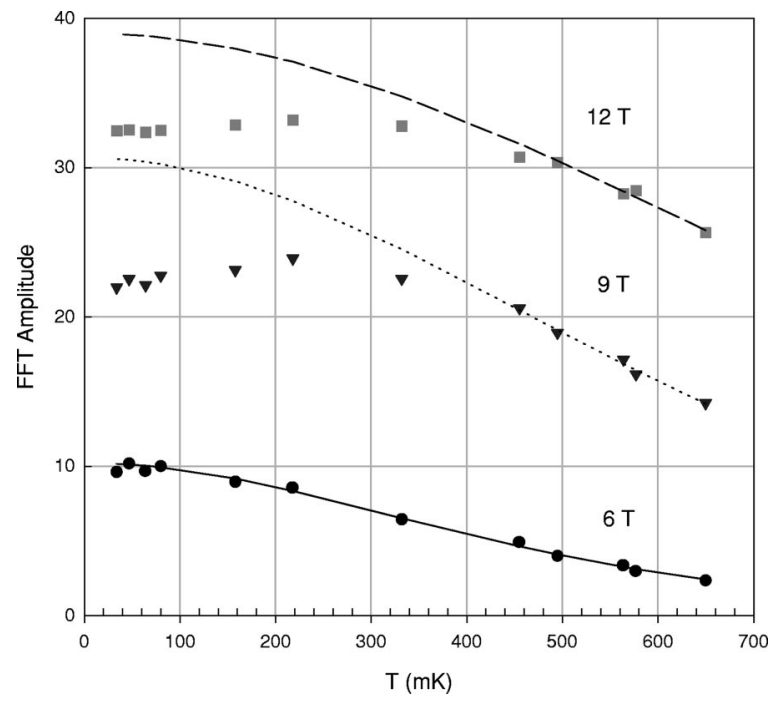

FIG. 4. Temperature dependence of the FFT amplitude at different fields. The lines are fits to LK theory. sponding higher temperatures. The Dingle temperature extrapolated from fitting the low field data gives a $T_{D}$ $\approx 0.5 \mathrm{~K}$. Similar results at high temperatures $(T>0.5 \mathrm{~K})$ and high fields (up to $30 \mathrm{~T}$ ) have been reported. ${ }^{14,15}$ At higher temperatures, the characteristic field, where deviation from the LK theory occurs, is higher. ${ }^{15}$ The difference between the FFT results and the relative changes shown in Fig. 2 is that the FFT amplitude is calculated for a field spread of $1 \mathrm{~T}$ around the target field, while the previous data were obtained by using the envelop calculations. Nevertheless, the systematic field and temperature dependence are the same for both approaches.

The deviation from the LK description indicates either the strong $2 \mathrm{D}$ nature of the compound studied or inappropriate analysis of the $\Delta \sigma / \sigma$. In comparison with other well studied 2D BEDT-TTF based salts, the present salt has one of the lowest ambient pressure effective mass of about $2 m_{e}$. The effective mass for $\kappa$-(BEDT-TTF) ${ }_{2} \mathrm{I}_{3}$ is $1.9 \mathrm{~m}_{e}$ for the small orbit and $3.9 m_{e}$ for the large orbit. $^{2}$ For $\kappa$-(BEDT-TTF $)_{2} \mathrm{Cu}(\mathrm{NCS})_{2}$, it is $3.5 m_{e}$ for the small orbit and $7 m_{e}$ for the large orbit. ${ }^{1}$ For $\kappa$-(BEDT-TTF $)_{2} \mathrm{Cu}\left[\mathrm{N}(\mathrm{CN})_{2}\right] \mathrm{Br}$, only $m \approx 6 m_{e}$ for the large orbit is found. ${ }^{6,8}$

The oscillation frequency is among the lowest with $F$ $=200 \mathrm{~T}$. In comparison, the $\alpha$ orbit has $F=598 \mathrm{~T}$ while $\beta$ orbit has $F=3800 \mathrm{~T}$ for the $\kappa$-(BEDT-TTF $)_{2} \mathrm{Cu}(\mathrm{NCS})_{2}$ salt and similar values of 570 and $3800 \mathrm{~T}$ for the two orbits for the $\kappa$-(BEDT-TTF) ${ }_{2} \mathrm{I}_{3}$ salt. This corresponds to a Fermi surface roughly three times smaller than that of the $\alpha$ orbit in the $\kappa$-phase salt. Note that the measured Fermi surface is approximately $1 / 5$ of that from the band structure calculation. ${ }^{10}$ More careful calculation using double-zeta Slater type orbitals may be necessary to verify the discrepancy. It should also be pointed out that very slow oscillations have been observed under pressure in the $\kappa$-(BEDT-TTF) ${ }_{2} \mathrm{Cu}_{2}(\mathrm{CN})_{3}$ (Ref. 16) and $\kappa$-(BEDT-TTF) ${ }_{2} \mathrm{Cu}\left[\mathrm{N}(\mathrm{CN})_{2}\right] \mathrm{Br}$ (Ref. 6,8) compounds accompanied by fast oscillations. However, there is no other oscillation frequency reported in the present compound for field up to $30 \mathrm{~T}$. $^{15}$

The origin of the insulating behavior is not clear. One possibility is that a density wave is developed at low temperatures and high fields. In the 2D $\alpha$-(BEDT-TTF $)_{2} M \mathrm{Hg}(\mathrm{SCN})_{4}$ with $M=\mathrm{K}, \mathrm{Rb}$, and $\mathrm{Tl}$, a spin density wave is presumably observed at $8-10 \mathrm{~K}$ with a corresponding hump in resistivity. ${ }^{3-6}$ At high fields, the spin density wave is suppressed and a field induced insulator-tometal transition has been observed. In a quasi-1D system such as (TMTSF) ${ }_{2} X$ with $X=\mathrm{PF}_{6}, \quad \mathrm{ClO}_{4}$, a field induced spin density wave has been observed. ${ }^{17,18}$ The effect of magnetic field is to reduce the dimensionality of the electronic system. Magnetic field forces the electrons moving along open orbits to oscillate in the perpendicular direction of finite width. With increasing field, the width decreases and the charges are moving along an increasingly more 1D orbit, thus leading to the formation of a density wave. Band structure calculations predict the presence of open orbits in the studied compound. ${ }^{14}$ With increasing field, it is possible that partial nesting can rise from the open bands, resulting in a semiconductinglike temperature dependence of the background magnetoresistance. In an ideal case the open bands contribute to the background magnetoresistance and the closed bands give rise to the $\mathrm{SdH}$ oscillation. The qua- 
dratic field dependence is generally consistent with the presence of open bands and this may also explain the normally large positive magnetoresistance observed in the $\alpha$-(BEDT-TTF $)_{2} M \mathrm{Hg}(\mathrm{SCN})_{4}, \kappa$-(BEDT-TTF $)_{2} \mathrm{Cu}(\mathrm{NCS})_{2}$, and $\kappa$-(BEDT-TTF $)_{2} \mathrm{I}_{3}$ salts. The absence of the semiconducting temperature dependence in the $\kappa$ salts may be related intrinsically to the feasibility of nesting of open bands. In the case of $\kappa$-(BEDT-TTF $)_{2} \mathrm{I}_{3}$, magnetic breakdown with an onset field of a few $\mathrm{T}$ due to the small energy gap between the closed and open orbits destroys any possible nesting. The absence of a thermally activated temperature dependence of the background resistance in the present compound suggests a partial nesting of the open bands, instead of a fully gaped state.

Another possibility is a field induced localization of charge carriers. ${ }^{19,20}$ Since electrons are strongly confined in the Landau tubes, scattering is necessary for charge conduction unlike the conventional metals. With increasing temperature, thermal scattering increases, thus leading to a smaller resistance. This is somewhat similar to the case of weak localization where thermally activated hopping is the dominating mechanism for conduction. While the argument should be true for the other salts, it is possible that the title compound has a much smaller Fermi surface. For example, at $18 \mathrm{~T}$, the highest occupied Landau orbits has $n=11$, while a similar field for the $\kappa$-phase salts will yield $n \simeq 33$. The small Landau index may be responsible for the pronounced localization effect. However, the temperature dependence of the background resistance is uncharacteristic of a weakly localized system. ${ }^{19,20}$

Because of the anomalous field and temperature dependence of the background magnetoresistance, the analysis of the $\Delta \sigma / \sigma$ in terms of LK theory is questionable, especially if there is an appreciable contribution of the open orbit to the conductivity. However, the fact that $\Delta \sigma / \sigma$ can be reasonably described by the LK theory at high temperature and low field suggests otherwise. The deviation from the LK model at low temperature and high field seems to be consistent with the 2D nature of the compound or a larger contribution from the open band at high field. Similar deviations have been observed in $\kappa$-(BEDT-TTF $)_{2} \mathrm{I}_{3}{ }^{2}$

In summary, we have reported a field induced metalinsulator transition in the all organic superconductor $\beta^{\prime \prime}$-(BEDT-TTF) ${ }_{2} \mathrm{SF}_{5} \mathrm{CH}_{2} \mathrm{CF}_{2} \mathrm{SO}_{3}$. The results are drastically different from the other BEDT-TTF based organic superconductors, where metallic temperature dependence of the background magnetoresistance has been observed. Analysis in terms of $\Delta \sigma / \sigma$ shows strong deviations from the LK description of the $\mathrm{SdH}$ oscillations at high field and low temperatures. The origin remains unclear. It is proposed that the background magnetoresistance may be related to the open band contribution and the nonmetallic nature could arise from partial nesting of the open orbits and consequently the formation of field induced density wave at low temperatures and high fields. Field induced localization is also a possible candidate for the observed anomalous magnetoresistance. Further investigation is necessary to clarify the mechanism.

This work was supported in part by NSF Grant No. DMR-9623306 and the Petroleum Research Fund ACS-PRF 33812-AC. Work performed at the National High Magnetic Field Laboratory was supported by the NSF, the state of Florida, and the U.S. Department of Energy. Work at Argonne National laboratory was supported by the U.S. Department of Energy, Office of Basic Energy Sciences, Division of Materials Sciences, under Contract No. W-31-109-ENG38. Work at Portland State University was supported by NSF Grant No. CHE-9632815 and the Petroleum Research Fund No. ACS-PRF 31099-AC1.
${ }^{1}$ K. Oshima, T. Mori, H. Inokuchi, H. Urayama, H. Yamochi, and G. Saito, Phys. Rev. B 38, 938 (1988).

${ }^{2}$ N. Harrison, C. H. Mielke, D. G. Rickel, J. Wosnitza, J. S. Qualls, J. S. Brooks, E. Balthes, D. Schweitzer, I. Heinen, and W. Strunz, Phys. Rev. B 58, 10248 (1998).

${ }^{3}$ T. Osada, R. Yagi, A. Kawasumi, S. Kagoshima, N. Miura, M. Oshima, and G. Saito, Phys. Rev. B 41, 5428 (1990).

${ }^{4}$ J. S. Brooks, C. C. Agosta, S. J. Klepper, M. Tokumoto, N. Kinoshita, H. Anzai, S. Uji, H. Aoki, A. S. Perel, G. J. Athas, and D. A. Howe, Phys. Rev. Lett. 69, 156 (1992).

${ }^{5}$ T. Sasaki and N. Toyota, Solid State Commun. 82, 447 (1992).

${ }^{6}$ M. V. Kartsovnik, A. E. Kovalev, V. N. Laukhin, S. I. Pesotskii, and N. D. Kushch, JETP Lett. 55, 39 (1992).

${ }^{7}$ M. V. Kartsovnik, G. Yu. Logvenov, H. Ito, T. Ishiguro, and G. Saito, Phys. Rev. B 52, R15 715 (1995); H. Weiss, M. V. KArtsovnik, W. Biberacher, E. Steep, A. G. Jansen, and N. D. Kushch, JETP Lett. 66, 202 (1997).

${ }^{8}$ C. H. Mielke, N. Harrison, D. G. Rickel, A. H. Lacerda, R. M. Vestal, and L. K. Montgomery, Phys. Rev. B 56, R4309 (1997).

${ }^{9}$ J. Wosnitza, Fermi Surfaces of Low-Dimensional Organic Metals and Superconductors (Springer, Berlin, 1996).

${ }^{10}$ U. Geiser, J. A. Schlueter, H. H. Wang, A. M. Kini, J. M. Williams, P. P. Sche, H. I. Zakowicz, M. L. VanZile, and J. D. Dudek, J. Am. Chem. Soc. 118, 9996 (1996); J. A. Schlueter, U.
Geiser, J. M. Williams, J. D. Dudek, M. E. Kelly, J. P. Flynn, R. R. Wilson, H. I. Zakowicz, P. P. Sche, D. Naumann, T. Roy, P. G. Nixon, R. W. Winter, and G. L. Gard, Synth. Met. 85, 1453 (1997).

${ }^{11}$ A. Audouard, P. Auban-Senzier, V. N. Laukhin, L. Brossard, D. Jerome, and N. D. Kushch, Europhys. Lett. 34, 599 (1996).

${ }^{12}$ X. Su, F. Zuo, J. A. Schlueter, J. M. Williams, P. G. Nixon, R. W. Winter, and G. L. Gard, Phys. Rev. B 59, 4376 (1999).

${ }^{13}$ D. Shoenberg, Magnetic Oscillations in Metals (Cambridge University Press, Cambridge, 1984).

${ }^{14}$ D. Beckmann, S. Wanka, J. Wosnitza, J. A. Schlueter, J. M. Williams, P. G. Nixon, R. W. Winter, G. L. Gard, J. Ren, and M.-H. Whangbo, Eur. Phys. J. B 1, 295 (1998).

${ }^{15}$ J. Wosnitza, S. Wanka, J. S. Qualls, J.S. Brooks, C. H. Mielke, N. Harrison, J. A. Schlueter, J. M. Williams, P. G. Nixon, R. W. Winter, and G. L. Gard, Synth. Met. 103, 2000 (1999).

${ }^{16}$ E. Ohmichi, H. Ito, T. Ishiguro, G. Saito, and T. Komatsu, Phys. Rev. B 57, 7481 (1998).

${ }^{17}$ L. P. Gor'kov and A. G. Lebed, J. Phys. (France) Lett. 45, L433 (1984).

${ }^{18}$ W. Kang, S. T. Hannahs, and P. M. Chaikin, Phys. Rev. Lett. 70, 3091 (1993), and references therein.

${ }^{19}$ P. A. Lee and T. V. Ramakrishnan, Rev. Mod. Phys. 57, 287 (1987).

${ }^{20}$ B. Kramer and A. MacKinnon, Rep. Prog. Phys. 56, 1469 (1993). 